\title{
Multifocal Tubercular Osteomyelitis Involving Bilateral Symmetrical Second Metacarpals
}

\author{
Pokharel R. \\ Dept. of Orthopaedics and Trauma Surgery, Institute of Medicine, Tribhuvan University, Kathmandu, Nepal
}

Correspondence address: Dr. Rohit Pokharel, Dept. of Orthopaedics and Trauma Surgery, Institute of Medicine, Tribhuvan University, Kathmandu, Nepal

\begin{abstract}
Skeletal tuberculosis involving small bones of the hand is less common.

We report a case, 6 year old boy, of bilateral symmetrical tubercular osteomyelitis of second metacarpals. He presented with gradually increasing swellings over bilateral hands over duration of one month. There was no other significant history relating to tuberculosis. Hand X-rays showed expansive lesions of bilateral second metacarpals. Thick pus and necrotic tissue was removed from both the swelling and from the bone. Presence of typical tubercular granuloma in the histopathological examination confirmed the diagnosis. The boy was treated with antitubercular drugs and improved after completion of anti-tubercular treatment.

Tuberculosis can present in different ways, and should be kept as one of the possible diagnosis of several bony pathologies including metacarpals and phalanges, especially in this part of the world where tuberculosis endemic.
\end{abstract}

Key word: tubercular dactylitis, metacarpals, symmetrical

\section{Introduction}

Extra-pulmonary tuberculosis is less common than the pulmonary form with an incidence of about $10 \%$, Spine is the most frequent site of skeletal involvement. 1,12 Tubercular infection of metacarpals, metatarsals and phalanges of hands and feet is known as tubercular dactylitis and it is uncommon especially after the age of five years. 1,8 Radiographic feature of cystic expansion of the short tubular bones in tubercular dactylitis is called "spina ventosa". , ,9,21 We report a rare case of multifocal tubercular osteomyelitis with symmetrical spina ventosa of bilateral second metacarpals of the hands.

Clinical record

A six years old boy, presented with swellings of bilateral hands which was gradually increasing in size over period of one month with minimal symptom of pain. It was associated with mild fever. He had an average height and built for his age, and had no previous co-morbid condition. His guardian did not give history of treatment of tuberculosis and had no family history of tuberculosis. The boy had received routine childhood vaccinations including BCG. On extermination his vitals were stable and there was no lymphadenopathy.

On examination of his hands, left hand had $3 \times 3.5 \mathrm{~cm}$ oval shaped swelling over the second metacarpal extending to the first web space with excoriated skin. On palpation local temperature was mildly raised with minimal tenderness. Consistency of the swelling was soft to firm. Right hand swelling was almost similar but less prominent with diffuse margins and more healthy skin over it (Figure 1).

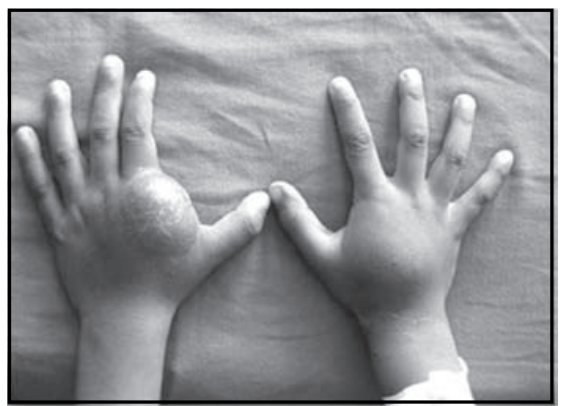

Fig 1: Photographs of bilateral hands showing swelling over 1st web space, and excoriation of skin on left hand. 
The wrist joints, metacarpo-phalangeal joints and interphalangeal joints were freely mobile. There was no neurovascular deficit in both the hands.

Routine haemogram showed; $12.8 \mathrm{gm} \%$ haemoglobin; total leukocyte count was $11,100 / \mathrm{cmm}$ with differential leukocyte count of neutrophils $51 \%$, lymphocytes $46 \%$, monocytes $02 \%$, eosinophils $01 \%$, and erythrocyte sedimentation rate was $20 \mathrm{~mm} / 1 \mathrm{st} \mathrm{hr}$. His random blood sugar level was $5.5 \mathrm{~m} \mathrm{~mol} / \mathrm{L}$. His routine urine analysis, renal function, serology for hepatitis and HIV were all normal. Mantoux test showed indurations of $22 \mathrm{~mm}$ at 72 hours (Figure 2). His chest X-ray was normal. X-rays of both hands, antero-posterior and oblique views showed expansive bone lesions in bilateral second metacarpals.

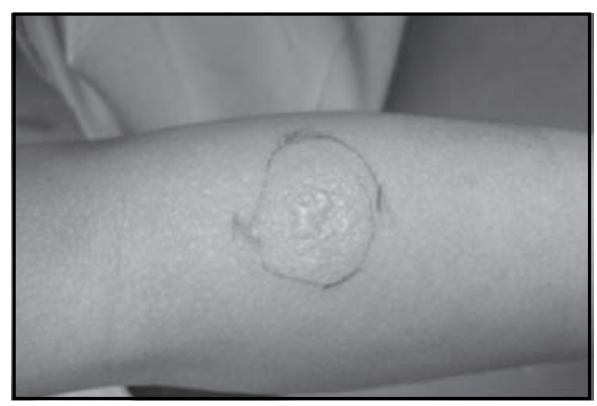

Fig. 2: Result of Montoux test, induration area after 72 hours.

In the left hand the lesion was at stage 3 (expansion of the second metacarpals with periosteal thickening, destruction of the bone with sequestrum) and in the right hand the lesion was at stage 2 (the metacarpal bone was swollen) 15 (Figure 3).

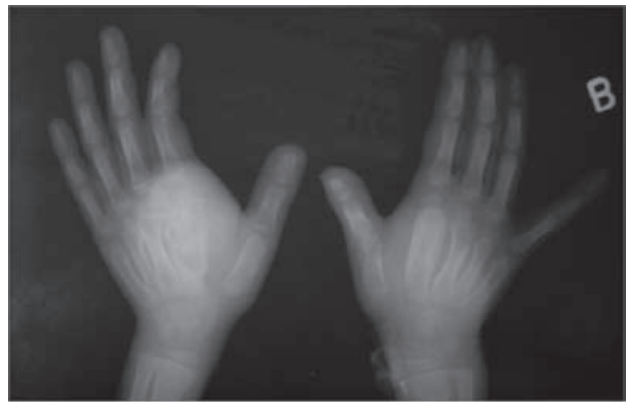

Fig. 3: X- Ray of bilateral hands AP view showing "spina ventosa" changes in second meta-carpals of both hands.
Thick pus was aspirated from left hand swelling and was sent for Gram's stain, AFB staining and for culture and sensitivity test. There was no growth in culture, both for tubercular and pyogenic bacteria. Gram Stain and AFB staining of the aspirate were negative.

He was admitted for drainage of pus and biopsy from the pathological sites, and was operated (Figure 4).

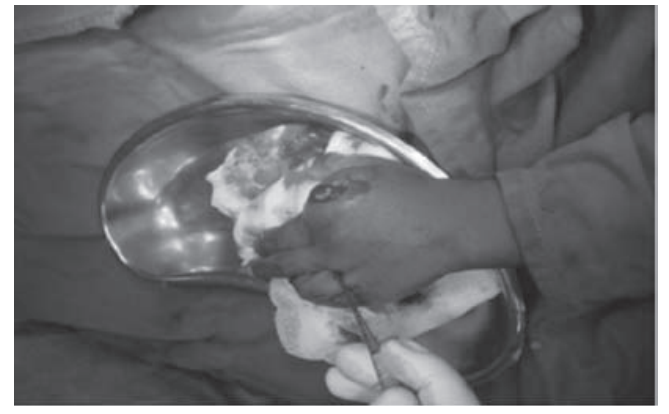

Fig. 4: Per-operative. Thick pus mixed with unhealthy granulation tissue.

During operation thick pus mixed with unhealthy granulation tissues and pieces of sequestra was removed without much disturbing the affected bones. His histopathological report showed typical features of tubercular granuloma (Figure 5).

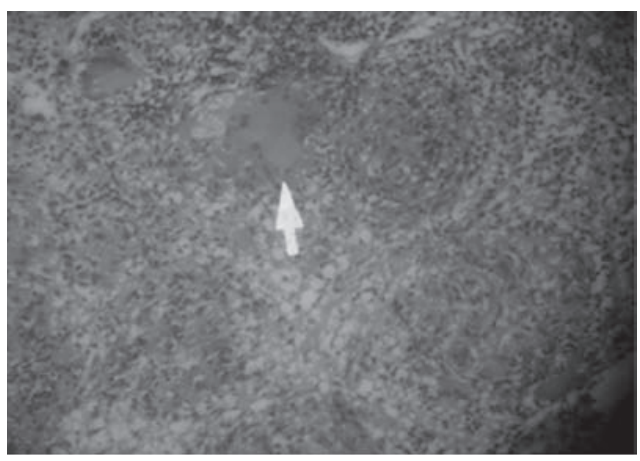

Fig. 5: Histopathological findings showing tubercular granuloma. Arrow shows area of area of casseation.

After establishment of diagnosis of tubercular osteomyelitis, anti-tubercular treatment was begun with 3 drugs (Rifampicin, Isoniazid and Pyrazinamide) for 3 months, followed by 2 drugs (Rifampicin, Isoniazid) for 9 months (total 12 months) as recommended by Nepal Orthopaedic Association.7 Ethambutol was not given as the case was from paediatric group. At 10th post operative day the swelling subsided, wound started healing and skin started to contract (Figure 6). 


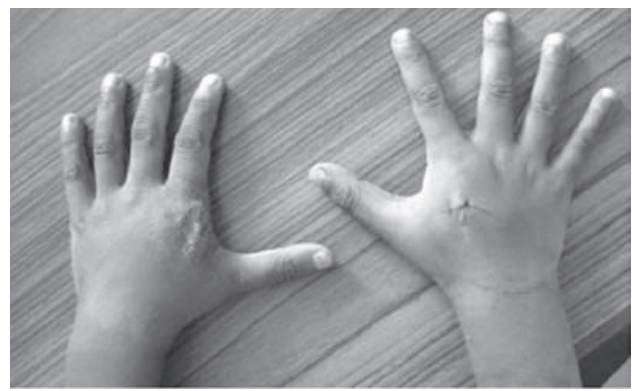

Fig. 6: Follow up visit on 10th post op day. Wound is healing well and size of the swellings regressing.

He was referred to local Directly Observed Therapy Short Course (DOTS) clinic for supply of the anti-tubercular drugs. According to the record of the DOTS clinic he completed the ATT course and had complete recovery from disease. However, he did not come for follow-up to our hospital.

\section{Discussion}

Bones and joints are affected in 1 to $3 \%$ of all cases tuberculosis, the spine and the hip joint involvement are most commonly involved, occupying more than $50 \%$ of them.1,2,5,22 It occurs in $1-5 \%$ of children who have untreated initial pulmonary tuberculosis. 6 Concomitant active pulmonary tuberculosis is less common and is present only in about $1 / 3$ rd of patients with skeletal tuberculosis. $3,6,13,14,30$ However, disseminated skeletal tuberculosis without primary foci is rare. 21

Tubercularinfection of themetacarpalsandphalangesisarare presentation of extra-pulmonary tuberculosis.1,2,6,13,24 The incidence of tubercular dactylitis is reported to be higher among the children compared to adults.4,10,14,28 The bones of the hands are more frequently affected than bones of the feet.10,14 There is a close association of tuberculosis with HIV/AIDS infection where skeletal tuberculosis can be disseminated with involvement of fingers.17,18 Rarely, multifocal tubercular dactylitis can occur without immunecompromise. 27

Diagnosis of tubercular dactylitis is based on the clinical picture and radiographic features, and should be confirmed by open biopsy and/or isolation of mycobacterium tuberculi in the culture.13,16

Insidious onset of swelling with mild or no pain and no pyrexia, usually without other constitutional features of tuberculosis is common presentation of tubercular dactylitis. This mild presentation of the disease condition makes diagnosis delay.9,13,14,15,16,18,20,26,28 Sometimes tubercular osteomyelitis can be masked by pyogenic osteomyelitis.23 Unlike in developed part of the world where there is lot of diagnostic dilemma due to rarity of the tubercular infection particularly when the presentation is unusual; tubercular dactylitis should be one of the probable differential diagnosis in any osseous pathology of hands and feet, particularly in countries where tuberculosis is endemic.5,6,7,8,10,12,13,14,16

Blood examination may show mild increase in erythrocyte sedimentation rate.13 Mantaoux test is usually positive. 16 Radiographs are still the mainstay of evaluation of patients with bony lesions.12 "Spina Ventosa" is radiological characteristics of tuberculous osteomyelitis.1,3,4,9,10 $, 11,12,14,28$ It typically involves short tubular bones of the hands and the feet in children. The hematopoietic marrow in the paediatric short bones offers a fertile field for hematogenous bacterial implantation and the infection rapidly spreads in the entire marrow space. The granulation tissue in the marrow expands the relatively soft cortex due to resorption or infarction by the underlying disease process. As a result, the involved bone resembles an inflated balloon; fusiform shape with thinned cortex and relatively radiolucent marrow space. Typically, there is no periosteal layering or thickening, and sequestration ordinarily does not occur. Sclerosis may be seen in long standing cases. $11,12,13,15,16$

Tubercular osteomyelitis of metacarpals and phalanges appear to be a sequential manifestation of the disease process, and 3 radiological stages has been described.15 In our case, left second metacarpal was at stage three and right second metatarsal was at stage two, which might be because of simultaneous infiltration of the bones by tubercular bacteria.

Similar lesions can be encountered in cases with dactylitis in sickle cell disease, congenital syphilis, pyogenic osteomyelitis, fungal infections, hereditary acro-osteolytic conditions, histiocytosis $\mathrm{X}$ and bone tumors. In sickle cell dactylitis, the feature is similar to that of tubercular dactylitis and is characteristically bilateral and dissolution of the sickle cell lesions is typically followed by irregularly sclerotic new bone formation. In syphilis, the bone is thickened by periosteal reaction. Clinically, pyogenic osteomyelitis tends to be acutely painful, swollen, and hot, with generalized fever.19 Tuberculous osteomyelitis is more often only mildly painful, pyrexia is minimal, and the whole condition is relatively benign. 14

Isolation of the mycobacterium tubercle bacilli from the tissue or discharge is the gold standard diagnostic tool.1,16,17,20 Presence of granulomatous osteitis with caseous necrosis in histo-pathological examination of 
Fig. 6: Follow up visit on 10th post op day. Wound is healing well and size of the swellings regressing.

He was referred to local Directly Observed Therapy Short Course (DOTS) clinic for supply of the anti-tubercular drugs. According to the record of the DOTS clinic he completed the ATT course and had complete recovery from disease. However, he did not come for follow-up to our hospital.

\section{Discussion}

Bones and joints are affected in 1 to $3 \%$ of all cases tuberculosis, the spine and the hip joint involvement are most commonly involved, occupying more than $50 \%$ of them.1,2,5,22 It occurs in $1-5 \%$ of children who have untreated initial pulmonary tuberculosis. 6 Concomitant active pulmonary tuberculosis is less common and is present only in about $1 / 3$ rd of patients with skeletal tuberculosis. $3,6,13,14,30$ However, disseminated skeletal tuberculosis without primary foci is rare. 21

Tubercularinfection of themetacarpals and phalangesisarare presentation of extra-pulmonary tuberculosis.1,2,6,13,24 The incidence of tubercular dactylitis is reported to be higher among the children compared to adults.4,10,14,28 The bones of the hands are more frequently affected than bones of the feet.10,14 There is a close association of tuberculosis with HIV/AIDS infection where skeletal tuberculosis can be disseminated with involvement of fingers.17,18 Rarely, multifocal tubercular dactylitis can occur without immunecompromise. 27

Diagnosis of tubercular dactylitis is based on the clinical picture and radiographic features, and should be confirmed by open biopsy and/or isolation of mycobacterium tuberculi in the culture. 13,16

Insidious onset of swelling with mild or no pain and no pyrexia, usually without other constitutional features of tuberculosis is common presentation of tubercular dactylitis. This mild presentation of the disease condition makes diagnosis delay.9,13,14,15,16,18,20,26,28 Sometimes tubercular osteomyelitis can be masked by pyogenic osteomyelitis.23 Unlike in developed part of the world where there is lot of diagnostic dilemma due to rarity of the tubercular infection particularly when the presentation is unusual; tubercular dactylitis should be one of the probable differential diagnosis in any osseous pathology of hands and feet, particularly in countries where tuberculosis is endemic.5,6,7,8,10,12,13,14,16

Blood examination may show mild increase in erythrocyte sedimentation rate. 13 Mantaoux test is usually positive. 16 Radiographs are still the mainstay of evaluation of patients with bony lesions.12 "Spina Ventosa" is radiological characteristics of tuberculous osteomyelitis.1,3,4,9,10 ,11,12,14,28 It typically involves short tubular bones of the hands and the feet in children. The hematopoietic marrow in the paediatric short bones offers a fertile field for hematogenous bacterial implantation and the infection rapidly spreads in the entire marrow space. The granulation tissue in the marrow expands the relatively soft cortex due to resorption or infarction by the underlying disease process. As a result, the involved bone resembles an inflated balloon; fusiform shape with thinned cortex and relatively radiolucent marrow space. Typically, there is no periosteal layering or thickening, and sequestration ordinarily does not occur. Sclerosis may be seen in long standing cases. $11,12,13,15,16$

Tubercular osteomyelitis of metacarpals and phalanges appear to be a sequential manifestation of the disease process, and 3 radiological stages has been described.15 In our case, left second metacarpal was at stage three and right second metatarsal was at stage two, which might be because of simultaneous infiltration of the bones by tubercular bacteria.

Similar lesions can be encountered in cases with dactylitis in sickle cell disease, congenital syphilis, pyogenic osteomyelitis, fungal infections, hereditary acro-osteolytic conditions, histiocytosis $\mathrm{X}$ and bone tumors. In sickle cell dactylitis, the feature is similar to that of tubercular dactylitis and is characteristically bilateral and dissolution of the sickle cell lesions is typically followed by irregularly sclerotic new bone formation. In syphilis, the bone is thickened by periosteal reaction. Clinically, pyogenic osteomyelitis tends to be acutely painful, swollen, and hot, with generalized fever.19 Tuberculous osteomyelitis is more often only mildly painful, pyrexia is minimal, and the whole condition is relatively benign. 14

Isolation of the mycobacterium tubercle bacilli from the tissue or discharge is the gold standard diagnostic tool.1,16,17,20 Presence of granulomatous osteitis with caseous necrosis in histo-pathological examination of a biopsy specimen is essential for confirmation of the diagnosis. $1,3,5,8,12,13,18,28$

Tubercular osteomyelitis of metacarpals has been reported either in single metacarpal5,8,9,14,16,25 or multiple metacarpals of the same hand along with other skeletal tuberculosis and pulmonary tuberculosis. 1,3,5,21,28 Disseminated tuberculosis with involvement of fingers can be seen in patients with HIV/AIDS.17 There are case reports on multifocal tuberculous dactylitis involving multiple phalanges of both hands 18 and chronic osteomyelitis in the second and third metacarpals of the left hand and arthritis 
of the left carpal joints.19 However, there has been no report in the literature, describing multifocal tubercular osteomyelitis lesion in bilateral symmetrical metacarpals in a child without any immune-compromise and primary pulmonary lesion. Though tuberculosis is one of the common diseases in Nepal, tubercular dactylitis is not commonly seen in routine practice. Here we have reported a rare case of multifocal symmetrical spina ventosa involving second metatarsals of both hands in six years old boy.

In endemic regions like Nepal29, the clinical features, radiological appearance, elevated ESR and positive Mantoux test are sufficient to diagnose tuberculosis and begin treatment. Similarly, in our case who presented with mild fever, slightly painful localized swellings over second metatarsals of hands, positive Mantoux test, classic radiological and histopathological findings, and response to anti-tubercular therapy confirmed the diagnosis.

There has been a controversy on dose, duration and number of anti-tubercular drugs combination for treatment of musculoskeletal tuberculosis. It is a common practice among the orthopaedic surgeons, to avoid recurrence, to prescribe anti-tubercular drugs for longer period of time at higher doses.1,7,13,14,18 There are few studies that define the optimal duration of treatment of skeletal tuberculosis involving the hand. Some experts favor a prolonged course of therapy to optimize post-treatment function. Others argue that the paucibacillary nature of the lesion make a 6-month treatment course appropriate.16,20 Based on the result of a study on practice of orthopaedic surgeons working at different parts of the country, and recommendations of the symposium on management of musculoskeletal tuberculosis, Nepal Orthopaedic Association has recommended 3 categories of musculoskeletal tubercular infections and has recommended the first line antitubercular treatment regime.7 Current recommendations for the treatment include a 2 month initial phase of Isoniazid, Rifampicin, Pyrazinamide, and Ethambutol followed by a 6 to 12 month regimen of Isoniazid and Rifampicin.30 Seven Turkish cases with tuberculosis of the metacarpals and phalanges were successfully treated with 4-drug regimen for 2 months, followed by a 2 -drug regimen for 10 months without recurrence. 13 Clinicians from different parts of India16,20,27 treated their cases with 4 drugs for 2 months and 2 drugs for 4 months (total 6 months) and had good results. However, there are reports on cases with recurrence of the disease and clinicians had to start second line anti-tubercular treatment.18 We treated our case with rifampicine, isoniazide and pyrazinamide for 3 months followed by rifampicine and isoniazide for 9 months.

In most of the cases, conservative treatment is enough for treatment of tubercular osteomyelitis.13,14,15,16,20 However, there are recommendations that debridement of the lesion can be helpful both for prompt tissue diagnosis and rapid recovery with anti-tubercular treatment.3,8,9,17,28 This was supported by our experience in our case, there was rapid recovery with anti-tubercular treatment after drainage of pus and granulation tissues.

Osseous tuberculosis is necessary to keep in mind when making the differential diagnosis of several osseous pathologies, especially in endemic parts of the world. A normal chest radiograph, the absence of other foci of active tuberculosis, or the absence of systemic symptoms does not exclude the possibility of bone tuberculosis. It has been said that 'tubercular infection can affect any tissue of the body and it can behave any way'. Our patient had bilateral symmetrical tubercular osteomyelitis of second metacarpal bones of hands. Thus this case has been reported to highlight another unusual manifestation of the common disease - tuberculosis.

\section{Reference}

1. Tuli, SM.; Tuberculosis of the skeletal system (bones, joints, spine and bursal sheaths). 3rd Edition, New Delhi, Jaypee Brothers Medical Publisher, 2004

2. Robins, RH.; Tuberculosis of the wrist and hand; Br J Surg.; 1967,54:211-8

3. Benkeddache, Y. and Gottesman, H.; Skeletal tuberculosis of the wrist and hand: a study of 27 cases; J Hand Surg Am.; 1982,7,593-600

4. Andronikou, S. and Smith B.; "Spina ventosa"-tuberculous dactylitis; Arch Dis Child.; 2002,86,206

5. Evanchick, CC., Davis, DE. and Harrington TM.; Tuberculosis of peripheral joints: an often missed diagnosis; J Rheumatol.; 1986,13,187-9

6. Daniel, TM. and Debanne, SM.; The serodiagnosis of tuberculosis and other mycobacterial diseases by enzyme-linked immunosorbent assay; Am Rev Respir Dis.; 1987,135,1137-51

7. Pokharel, RK.; Anti-tubercular treatment regime for Musculoskeletal Tuberculosis; J Nepal Med Assoc.; 2006,45,279-80

8. Karanas, YL. and Yim, KK.; Mycobacterium tuberculosis infection of the hand: a case report and review of the literature; Ann Plast Surg.; 1998,40,65-7

9. Weber, P. and Rösslein R.; Rapidly growing tumor of the hand--is tuberculosis as differential diagnosis gaining increased importance?; Handchir Mikrochir 
Plast Chir. 1994,26,91-4

10. Salimpour, R. and Salimpour P.; Picture of the month. Tuberculous dactylitis; Arch Pediatr Adolesc Med.; 1997,151,851-2

11 Putschar, WGJ.; The skeletal pathology of tuberculosis; In: Taveras JM, Ferrucci JT: Radiology Diagnosis-Imaging-Intervention, Vol. 5 Philadelphia, Lippincott - Raven, 1996

12. Teo, HE, and Peh, WC.; Skeletal tuberculosis in children; Pediatr Radiol.; 2004,34,853-60

13. Subasi, M., Bukte, Y, Kapukaya, A. and Gurkan, F.; Tuberculosis of the metacarpals and phalanges of the hand; Ann Plast Surg.; 2004,53,69-72.

14. Dhillon, MS, Sharma, S, Gill, SS. and Nagi ON.; Tuberculosis of bones and joints of the foot: an analysis of 22 cases; Foot Ankle; 1993,14,505-13.

15. Agarwal, A, Qureshi, NA, Kumar, P. and Khan, S.; Tubercular osteomyelitis of metacarpals and phalanges in children; Hand Surg.;2011,16,19-27.

16. Kushwaha, RA, Kant, S, Verma, SK, Sanjay and Mehra, S.; Isolated metacarpal bone tuberculosis-a case report; Lung India; 2008,25,17-9.

17. Agarwal, S, Caplivski, D. and Bottone EJ.; Disseminated tuberculosis presenting with finger swelling in a patient with tuberculous osteomyelitis: a case report; Ann Clin Microbiol Antimicrob; 2005, $3,4: 18$

18. Hassen-Zrour, S, Younes, M, Haj Salah-Othman, M, Korbäa, W, Touzi, M, Béjia, I, Bergaoui, N and Hamdi, MF.; Multifocal tuberculous dactylitis: a case report; Chir Main.; 2008,27,122-5

19. Amine, B, Benbouazza, K, Harzy, T, Rahmouni, R and Hajjaj-Hassouni, N,; Chronic osteomyelitis of the metacarpals. Report of a case; Joint Bone Spine; 2005,72,322-5

20. Mishra, G, Dhamgaye, TM, and Fuladi, B.; Spina ventosa discharging tubercle bacilli- A case report; Amol Indian J Tuberc.; 2009,56,100-103

21. Kothari, PR, Shankar, G, Gupta, A, Jiwane, A. and Kulkarni, B.; Disseminated spina ventosa; Indian J Chest Dis Allied Sci.; 2004,46,295-6

22. Almeida, A.; Tuberculosis of the spine and spinal cord; Eur J Radiol.;2005,55,193-01

23. Yahia Zayani, CB, Daoued, L, Tekaya, R, bouebdella, M, Abdelmoula, L, Kooli, M, Chaabouni, L. and
Zouari, R.; Tuberculous osteomyelitis masked by staphylococcal infection; Joint Bone Spine.; 2009,76,429

24. Stanhope, B. and Dieppe, C.; Dactylitis-an uncommon presentation of tuberculosis; Pediatr Emerg Care; 2007,23,394-6

25. Rieger, H, Pennig, D, Edel, G. and Brug, E.; Tuberculosis of the hand; Handchir Mikrochir Plast Chir.; 1990,1,22,183-90

26. Herzog, A.; Dangerous errors in the diagnosis and treatment of bony tuberculosis; Dtsch Arztebl Int.; 2009, 106,573-7

27. Chowdhary, V, Aggarwal, A. and Misra, R.; Multifocal tubercular dactylitis in an adult; J Clin Rheumatol.; 2002,8,35-7

28. Malik, S, Joshi, S. and Tank, JS.; Cystic bone tuberculosis in children--a case series; Indian J Tuberc.; 2009,56,220-4

29. Massachusetts department of Public Health Bureau of Communicable of Disease, Division of Tuberculosis prevention and control. http:// www.who.int/tb/publications/global_report/2007/xls/ global.xls

30. Center for Disease Control and Prevention. Treatment of Tuberculosis. MMWR Morb Mortal Wkly Rep. pp. 1-77. June 20, 2003. 